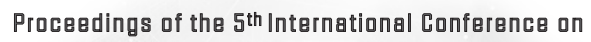

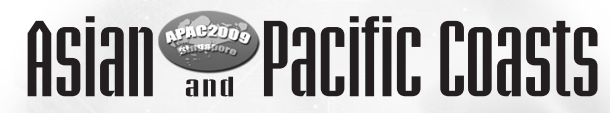


This page intentionally left blank 
Volume 2

Proceedings of the 5th International Conference on

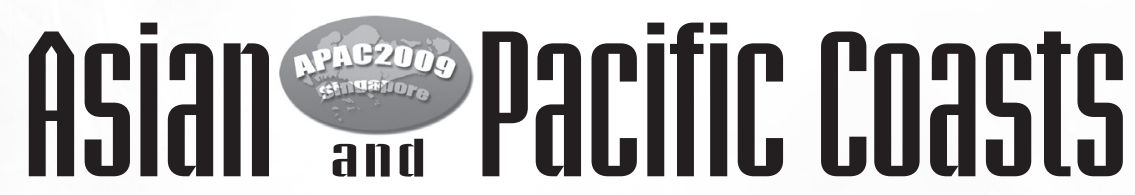

Singapore, $13-16$ Dctober 2009

Editors

\section{Soon Keat TAN \& Zhenhua HUANG}

Nanyang Technological University, Singapore

Organized by:

Maritime Research Centre and DHI-NTU Centre, NTU 
Published by

World Scientific Publishing Co. Pte. Ltd.

5 Toh Tuck Link, Singapore 596224

USA office: 27 Warren Street, Suite 401-402, Hackensack, NJ 07601

UK office: 57 Shelton Street, Covent Garden, London WC2H $9 \mathrm{HE}$

British Library Cataloguing-in-Publication Data

A catalogue record for this book is available from the British Library.

\section{ASIAN AND PACIFIC COASTS 2009 \\ (In 4 Volumes, with CD-ROM) \\ Proceedings of the 5th International Conference on APAC 2009}

Copyright (c) 2010 by World Scientific Publishing Co. Pte. Ltd.

All rights reserved. This book, or parts thereof, may not be reproduced in any form or by any means, electronic or mechanical, including photocopying, recording or any information storage and retrieval system now known or to be invented, without written permission from the Publisher.

For photocopying of material in this volume, please pay a copying fee through the Copyright Clearance Center, Inc., 222 Rosewood Drive, Danvers, MA 01923, USA. In this case permission to photocopy is not required from the publisher.

ISBN-13 978-981-4287-94-4

ISBN-10 981-4287-94-6 (Set) (pbk)

ISBN-13 978-981-4287-97-5 (Vol. 2) (pbk)

ISBN-10 981-4287-97-0 (Vol. 2) (pbk)

Printed in Singapore. 


\section{PREFACE}

The coastal zone has always been an important frontier - for trade, food and foundation for modern civilization. This same zone has also been exploited in one way or another and sometimes without regards to the nature's balance and scheme of things. It is only when somethings go terribly wrong that we begin to react and attempt to undo the mistakes of the past. At times we have succeeded, but at a high price. At times, we have to retreat and concede defeat as the technology of man is no match to the force of nature. Overtimes we learn to work with nature and leverage on science of nature to protect the coastal zone and hold our frontier between the sea and land.

We are now in the exciting and challenging era of climate change. The immediate future foretells higher seawater level, stronger winds, waves and currents. Globally we see increasing frequency of storm surges, increase intensity of rainfall and flood. We need to work together to advance the science and knowledge of the coastal zone. Scientists, engineers, and professionals need to work together to deal with coastal zone issues and challenges.

This set of four-volume proceedings collects all the papers presented at the $5^{\text {th }}$ International Conference on Asian and Pacific Coasts and covers a wide range of topics, including coastal and harbor structures, sediment processes and shoreline changes, coastal environmental problems and marine ecology, coastal zone management and planning, tsunami, ocean energy, global environmental problems, laboratory and field measurement techniques, numerical simulations of coastal problems, and basic research in ocean waves, currents, and tides. The proceedings will be a valuable reference book for researchers, engineers, professionals, and postgraduate students working on the coastal engineering problems. 
This page intentionally left blank 


\begin{abstract}
APAC Council
Chair Goda, Yoshimi (Coastal Development Institute of Technology, Japan)

Choi, Byung Ho (Sungkyunkwan University, Korea)

Mizuguchi, Masaru (Chuo University, Japan)

Qiu, Dahong (Dalian University of Technology, China)

Xie, Shileng (First Design Institute of Navigation Engineering, China)
\end{abstract}

\title{
International Steering Committee
}

Cheng, Liang (The University of Western Australia, Australia)

Choi, Byung Ho (Sungkyunkwan University, Korea)

Huang, Zhenhua (Nanyang Technological University, Singapore)

Kioka, Wataru (Nagoya Institute of Technology, Japan)

Lee, Joseph Hun-Wei (Hong Kong University, Hong Kong, China)

Li, Yucheng (Dalian University of Technology, China)

Lin, Ming-Chung (National Taiwan University, Taipei, China)

Liu, Philip L.F. (Cornell University, USA)

Nadaoka, Kazuo (Tokyo Institute of Technology, Japan)

Ou, Shan-Hwei (National Cheng Kung University, Tainan, China)

Pyun, Chong Kun (Myongji University, Korea)

Shibayama, Tomoya (Yokohama National University, Japan)

Suh, Kyung-Duck (Seoul National University, Korea)

Sundar, Vallam (Indian Institute of Technology, Madras, India)

Yamashita, Takao (Hiroshima University, Japan)

Yu, Xiping (Tsinghua University, China)

Zuo, Qihua (Nanjing Hydraulic Research Institute, China)

\section{APAC 2009 Local Organising Committee}

Soon Keat TAN (Nanyang Technological University) Chair

Eng Soon CHAN (National University of Singapore) Co-Chair

Zhenhua HUANG (Nanyang Technological University) Secretary-General

Nian Sheng CHENG (Nanyang Technological University)

Joy, CHUA L.H. (DHI-NTU Centre, Singapore)

Selina LEE (Maritime and Port Authority of Singapore) 


\section{APAC 2009 International Scientific Committee}

Liang CHENG (The University of Western Australia, Australia)

Yee Meng CHIEW (Nanyang Technological University, Singapore)

Mohamed S. GHIDAOUI (Hong Kong University of Science and Technology, Hong Kong)

Zhenhua HUANG (Nanyang Technological University, Singapore), Secretary

Ole LARSEN (DHI-NTU Centre, Singapore)

Adrian, LAW Wing-Keung (Nanyang Technological University, Singapore)

Pengzhi LIN (Sichuan University, China)

Cheng LIU (International Research and Training Centre on Erosion \& Sedimentation, China)

Hua LIU (Shanghai Jiaotong University, China)

Philip, LIU L.F. (Cornell University, USA)

Edmond, LO Yat-Man (Nanyang Technological University, Singapore)

Kyung Duck SUH (Seoul National University, Korea)

Yoshimitsu TAJIMA (The University of Tokyo, Japan)

Soon Keat TAN (Nanyang Technological University, Singapore)

Wataru KIOKA (Nagoya Institute of Technology, Japan)

Xiping YU (Tsinghua University, China) 


\section{CONTENTS}

Preface $\quad v$

Committee $\quad$ vii

Applicability of Sediments Excavated in River Channel to Beach Nourishment

Considering Impact on Habitat

Keisuke Murakami, Takeshi Ono

Observations of Fine Sediment Transport Event during Typhoon Attack in

Tokyo Bay

Yasuyuki Nakagawa, Ryuich Ariji, Shoichi Matsuzaka, Kazunobu Morohoshi,

Hiroshi Yagi, Kazuo Nadaoka, Susumu Tanaka

Observations of Suspended Sediment Properties off the Tenryu River Mouth, Japan

Syamsidik, Shigeru Kato, Shin-Ichi Aoki

Adaptability Research of Tidal Flat Reclamation and Tidal Inlet Stability in

Jiangsu Coastal Zone

Gong Zheng, Zhang Changkuan, Tao Jianfeng

Modeling of Wave-Current-Sediment and Seabed Evolution for Shanghai

Nanhuizui Inning Project

Bei Li, Zhixia Zhu

An Effect of Pore Water Infiltration Flow on Re-Suspension of Organic Mud Focus on the Effect of Pore Water Infiltration Flow

Kyung-Hoi Kim, Masami Abe, Tadashi Hibino, Katsuaki Komai

A Prediction for Sand Bar Formation at the Mouth of Takase Flood Diversion

Channel, Japan

Nguyen Trong Tu, Hitoshi Tanaka

Prediction of the Siltation Using Water Depth Monitoring in the Intake

Bumshick Shin, Kyuhan Kim

A Review of the Current State-of-the-Arts on the Application of Silt Screens as Sediment Control Equipment in Open Water

Vu Thu Trang, Tan Soon Keat 
On the Mechanism of Organic-Rich Sediment Accumulation at the Head of Tokyo Bay

Jun Sasaki, Yuta Sato, Thamnoon Rasmeemasmuang, Tomoya Shibayama

Sediment Transport and Morphodynamics of My A Inlet, Vietnam, in the Low Flow Season

Nghiem Tien Lam, Le Dinh Thanh, Vu Minh Cat, Vu Thi Thu Thuy

Deducing Past Coastal Conditions Using Old Photographs and Creation of Predicted Image of Coast

Toshinori Ishikawa, Takaaki Uda, Genji Aoshima, Atsushi Yoshioka

Quantification of In Situ Coastal Surfers based on Video Monitoring

Haijiang Liu, Hideo Funayama, Yoshimitsu Tajima, Shinji Sato

Longshore Structure of Wave Run-Ups under Different Stormy Conditions

Observed with X-Band Radar

G.M. Jahid Hasan, Satoshi Takewaka

An Improvement of Modified Bayesian Method for Estimating Directional Wave Spectra from HF Radar Backscatter

Lukijanto, Noriaki Hashimoto, Masaru Yamashiro

Development of a New Quantitative Monitoring System for Nearshore

Dynamics Using Video Images

Makoto Arii, Yoshimitsu Tajima

Fine-Scale Resolution Coastal Topographic and Bathmetric Mapping

Jinah Kim, Jae Seol Shim, Seonjeong Kim, Jinyoung Jeong, Hak Soo Lim

Water Resources Available in Australian's Coastal Zones and Its Development by Coastal Reservoirs

Shu-qing Yang

Community-Based Coastal Planning for Conservation of Habitat of Endangered Species and Biodiversity in Nakatsu Tidal Flat, Japan

Satoquo Seino, Takaaki Uda, Yukiko Ashikaga

Analysis of Effects of Reservoir Dam of Marsh of Jiaozhou Bay on Marine

Dynamic and Sedimentation Environment

Bingchen Liang, Dong Liu, Xiuli Lv, Xueqing Zhang 
Predicting Trends in Water Quality in the Coastal Zone of Tt-Hue, Vietnam An Assessment of Impacts of Rice Culture and Aquaculture

N.T.T. Nguyen, M.B. de Vries

Evolution of the Yangtze River Estuary and Regulation of the Xuliujing-Node

Xia Yimin, Qu Hongling

Sediment Treatment Research of Port and Waterway Engineering on

Silt Coast - Sediment Research Summary of Tianjin Port

Sun Lian-Cheng, Chen Chun

Estimating the Indirect Costs of Non Adapting Infrastructure to an Increase in Typhoon Intensity in the Asia-Pacific Region

Miguel Esteban, Christian Webersik, Tomoya Shibayama

Survival Analysis of Coastal Facilities

Hajime Tanji, Kiri Hiroride, Nakaya Tetsuo

Integrated Coastal Environmet Information Service System Using Web-GIS

Jinah Kim, Chang S. Kim, Hak Soo Lim, K.S. Park

Disaster Survey after the Cyclone Nargis in 2008

Tomoya Shibayama, Hiroshi Takagi, Ngun Hnu

Asian and Oceanian Coastal-Risk Projection due to Sea-Level Rise and Population Growth

Hisamichi Nobuoka, Nobuo Mimura, Makoto Tamura

Probabilistic Design and Reliability Analysis of Coastal Structures - A

Vietnam Case

Cong- Mai Van, Hung- Nguyen Quang, Han Vrijling, P.H.A.J.M. van Gelder

Light-Transmission Measurement of Sediment Concentration Based on Image Analysis

Takenori Shimozono, Akio Okayasu

Influence of Oceanic Water Intrusion on Coastal Environments in Winter around the Tama River Estuary in Tokyo Bay

Hiroshi Yagi, Tanuspong Pokavanich, Kazuo Nadaoka, Ryuichi Ariji,

Ken Furudoi, Hiroyuki Geshi, Taro Furudono, Yukimasa Oono

Modeling Mixing of Cooled Water Discharge by LNG Terminal

Il Won Seo, Chang Geun Song, In Ok Jun, Seung Yong Oh 
Roles of Sporadic Intrusion of Outer Sea Water in Water Exchange and Water

Quality Variations in the Coastal Lagoon of Puerto Galera, Philippines

Tanuspong Pokavanich, Kazuo Nadaoka, Ariel C. Blanco

Simulation of Saline Water Intrusion into Lower Part of Ca River and Solutions

to Mitigate Economic Losses in Dry Season

Vu Minh Cat, Vu Minh Duc

Quadtree Mesh for Combined Hydrodynamic and Water Quality Modelling Nguyen Quang Chien, Tan Soon Keat

Wavelet Multiresolution Signal Decomposition on Turbulent Wakes Generated by an Inclined Cylinder

S.F. Mohd. Razali, T. Zhou, L. Cheng

Dependence of Vortex Shedding on Inclination of a Stationary Cylinder

Tongming Zhou, S.F. Mohd. Razali, Liang Cheng

Experimental Studies of Vortex-Induced Vibrations on a Bundled Cylinder System Tongming Zhou, A. Lalli

Pattern Analysis for the Vortex Induced Vibration of an Inclined Cylinder

DongHo Jung, Deoksoo Moon, SeungWon Lee

Experimental Study of Flow about a Circular Cylinder in Shallow Water

Wang Xikun, Hao Zhiyong, Tan Soon Keat

Experimental Studies of the Vorticity Field in the Wake of Side-by-Side

Circular Cylinders

Hao Zhiyong, Zhou Tongming, Tan Soon Keat, Zhang Weiguo

Multi-Station Observations of the High Swell along the East Coast of Korea

Weon Mu Jeong, Sang-Ho Oh, Kyung-Ho Ryu, Dong Young Lee, Jang Won Chae

Field-Based Data Collection Techniques and Remote Sensing for Developing a High Resolution Digital Elevation Model for Coastal Studies

K. Santosh, D.K. Raju, J. Chandrasekar, Teh Tiong Sa

Morphological Monitoring of Coastal Dune Using Differential GPS and 3D

Terrestrial Laser

Junaidi, Naoto Wakae, Shigeru Kato, Shin-Ichi Aoki 
Introduction to Structural Health Monitoring System on "Uldolmok" Tidal Current Power Plant

Jin-Hak Yi, Woo-Sun Park, Jin-Soon Park, Kwang-Soo Lee

Potentiality of Bathymetry Monitoring Using Fish Finder Data for Sediment Management in a Wide Area

Takumi Okabe, Shin-Ichi Aoki, Shigeru Kato

Author Index

Appendices are found in the CD 